DOI: $10.1002 /$ cbic.201800078

Communication

\title{
Nanodisc-Targeted STD NMR Spectroscopy Reveals Atomic Details of Ligand Binding to Lipid Environments
}

Juan ${ }^{\wedge \wedge}$ C. Muñoz-García, ${ }^{[a]}$ Rosana Inacio ${ }^{\wedge \wedge} \operatorname{dos}^{\wedge \wedge}$ Reis,${ }^{[a]}$ Richard ${ }^{\wedge \wedge}$ J. Taylor,${ }^{[b]}$ Alistair ${ }^{\wedge \wedge} \mathrm{J}$. Henry, ${ }^{[b]}$ Anthony Watts 0000-0003-4976-1102*[a]

[a] $\quad<?><?>$ please give academic titles of all authors $<?>\langle ?\rangle$ <orgDiv/>Biomembrane Structure Unit $<$ orgDiv/>Department of Biochemistry, <orgName/>University of Oxford <street/>South Parks Road, <postCode/>OXI 3QU, <city/>Oxford (<country/>UK) E-mail: anthony.watts@bioch.ox.ac.uk. $<$ orgName/>UCB Celltech <street>216 Bath Road, <city/>Slough <postCode/>SL1 4EN (<country/>UK)

<pictid> Supporting information and the ORCID identification numbers for the <->authors of this article can be found under <url>https://doi.org/10.1002/cbic.201800078</url>.

How do ligands communicate with lipids? Nanodiscs are ideal nanoplatforms for STD NMR assessment of molecular recognition between small molecules and lipids. It was found that the neurotransmitter dopamine can only establish communication with negatively charged lipid environments. MSP: membrane scaffold protein, POPG: hexadecano-1-yl-2-(9Zoctadecenoyl)-sn-glycero-3-phospho-(1'-rac-glycerol).

Nanodisc-targeted STD NMR spectroscopy reveals atomic details of ligand binding to lipid environments (Watts@UniofOxford)

dopamine

lipids

nanodiscs

NMR spectroscopy

STD NMR spectroscopy 
Saturation transfer difference (STD) NMR spectroscopy is one of the most popular ligandbased NMR techniques for the study of protein--ligand interactions. This is due to its robustness and the fact that it is focused on the signals of the ligand, without any need for NMR information on the macromolecular target. This technique is most commonly applied to systems involving different types of ligands (e.g., small organic molecules, carbohydrates or lipids) and a protein as the target, in which the latter is selectively saturated. However, only a few examples have been reported where membrane mimetics are the macromolecular binding partners. Here, we have employed STD NMR spectroscopy to investigate the interactions of the neurotransmitter dopamine with mimetics of lipid bilayers, such as nanodiscs, by saturation of the latter. In particular, the interactions between dopamine and model lipid nanodiscs formed either from charged or zwitterionic lipids have been resolved at the atomic level. The results, in agreement with previous isothermal titration calorimetry studies, show that dopamine preferentially binds to negatively charged model membranes, but also provide detailed atomic insights into the mode of interaction of dopamine with membrane mimetics. Our findings provide relevant structural information for the design of lipid-based drug carriers of $\langle$ ? $><$ ? $>$ dopamine and its structural analogues $O K$ ? $\langle$ ? $>\langle ?$ ? $>$ and are of general applicability to other systems.

Saturation transfer difference (STD) NMR spectroscopy is a widely employed NMR technique for the investigation and characterisation of ligand binding to macromolecular receptors of different nature. STD NMR spectroscopy relies on the intermolecular fast exchange between a small molecule and a macromolecular entity, so that the characteristic negative nuclear Overhauser effect (NOE) of the macromolecule develops on the small ligand. Thus, it is particularly suited for the study of protein-carbohydrate interactions. ${ }^{[1-3]}$ It has been frequently used to investigate ligand binding to DNA and RNA oligonucleotides; ${ }^{\left[4,{ }^{\wedge}\right]}$ recently, it has also been applied to the study of ligand binding to membrane proteins. ${ }^{[6]}$

The first description of the thermodynamics of dopamine interactions with biomembranes was reported a few years ago. ${ }^{[7]}$ In particular, large unilamellar vesicles (LUVs) of 1,2-dimyristoyl-sn-glycero-3-phosphocholine (DMPC), 1,2-ditetradecanoyl-sn- 
glycero-3-phospho-(1'-rac-glycerol) (DMPG), and different mixtures of these two lipids were studied as model membranes by using isothermal titration calorimetry (ITC) and differential scanning calorimetry. Dopamine showed preferential binding to PG over PC lipids, with the hydrophobic contributions being ten times weaker than electrostatic forces. It was thus suggested that dopamine interacts superficially with the PG phospholipid head group without penetrating into the bilayer hydrocarbon core. However, information at the atomic level on dopamine interactions with model membranes is still absent, thus precluding any detailed understanding of dopamine's mode of binding to negatively charged membranes.

We have designed lipid nanodiscs to investigate the molecular recognition of dopamine to lipids 1-palmitoyl-2-oleoyl-sn-glycero-3-phosphocholine (POPC), hexadecano1-yl-2-(9Z-octadecenoyl)-sn-glycero-3-phosphoethanolamine (POPE), and hexadecano-1-yl2-(9Z-octadecenoyl)-sn-glycero-3-phospho-(1'-rac-glycerol) (POPG). The use of single-lipid nanodiscs of different head-group type, that is, zwitterionic non-hydrogen-bond-donor POPC, zwitterionic hydrogen-bond-donor POPE and negatively charged POPG allowed us to mimic biological membranes of different surface properties in a controlled manner. Following this approach, we have identified the lipid selectivities of dopamine--lipid interactions by using nanodisc-targeted STD NMR spectroscopy.

\section{Nanodiscs}

Size-exclusion chromatography (SEC) of POPC and POPG nanodisc samples showed a symmetric sharp peak centred around $12.5^{\wedge \wedge} \mathrm{mL}$ in both cases (Figure ${ }^{\wedge \wedge} 1<$ figr $1>$ ). This corresponds to a hydrodynamic radius of approximately $5^{\wedge \wedge} \mathrm{nm}\left(\approx 165^{\wedge \wedge} \mathrm{kDa}\right)$, as previously reported for nanodiscs assembled by using the membrane scaffold protein (MSP) 1D1 construct. ${ }^{[8]}$ The amount of discs obtained (area under the peaks) was very similar for both lipid types. No eluate corresponding to aggregated species was observed, thus indicating that the MSP-to-lipid ratio employed was optimally chosen to yield a monodisperse population of nanodiscs.

On the other hand, POPE nanodiscs showed an heterogeneous size distribution in the SEC profile. As for POPC and POPG nanodiscs, only the SEC fraction centred around $12.5^{\wedge \wedge} \mathrm{mL}$ was used for NMR characterisation. 


\section{NMR spectroscopy}

STD NMR experiments were carried out at high field (600 or $\left.750^{\wedge \wedge} \mathrm{MHz}\right)$, monitoring the evolution of STD signals by varying the saturation time $\left(t_{\text {sat }}\right)$. Figure ${ }^{\wedge} 2<$ figr $2>$ shows the STD NMR spectrum at $4^{\wedge \wedge}$ s saturation time for POPC- and POPG-only nanodiscs in the presence of dopamine. The data show that, whereas the anionic POPG nanodiscs give rise to intense dopamine STD signals for both the aromatic and aliphatic protons, the zwitterionic non-hydrogen-bond-donor POPC lipids do not interact with dopamine or do so to a much lower extent (no aliphatic and negligible aromatic STD signals are observed). Like POPC, when the same experiments were carried out with zwitterionic hydrogen-bond-donor POPE lipids, no STD signal was detected (Figure^^3 $<<$ figr $3>$ ). Thus, our results suggest that the driving force of dopamine binding to POPG nanodiscs is the negatively charged PG head groups, and not the rest of the lipid chain (or MSP acting as an interacting partner). In addition, we show for the first time that dopamine does not bind POPE lipids. To further confirm the lack of non-specific interactions of dopamine to MSP, a control STD experiment in the presence of $25^{\wedge} \wedge \mu \mathrm{M}$ MSP (no lipid) was performed; no dopamine resonances were observed.

A previous study of dopamine interactions to model PC (DMPC) and PG (DMPG) biomembranes by ITC showed the absence of binding to PC unilamellar vesicles, whereas binding affinity increased with increasing PG content in DMPG vesicles. Importantly, the observation of dopamine binding to POPG nanodiscs by STD NMR spectroscopy indicates that the affinity of interaction is low $\left(K_{\mathrm{d}} \approx \mathrm{mM}--\mu \mathrm{M}\right)$, that is, fast-exchange conditions on the NMR timescale. This is supported in the same ITC study, in which an affinity constant of $705^{\wedge} \mathrm{M}^{<\mathrm{M}->1}$ (i.e., $K_{\mathrm{D}} \approx 1.4^{\wedge \wedge} \mathrm{mM}$ ) for dopamine binding to DMPG vesicles was reported. ${ }^{[7]}$

To understand the molecular recognition between dopamine and PG lipids, STD NMR spectroscopy experiments were carried out at different saturation times from $0.5^{\wedge \wedge} \mathrm{s}$ to $6^{\wedge \wedge} \mathrm{s}$. This procedure allowed us to obtain the so-called STD build-up curves for dopamine binding to POPG nanodiscs, and, subsequently, derive their initial slope and normalise all the STD values against the highest one (H5, Figure ${ }^{\wedge \wedge} 4<$ figr $\left.4>\right)$. Thus, we were able to map the main contacts of dopamine to PG lipids in the bound state (binding epitope) at high resolution 
(Figure ${ }^{\wedge} 4<x$ figr $4>$ ). It should be noted that the use of STD initial slopes increases the accuracy of the method by avoiding the detrimental effects of the different relaxation properties of the ligand protons on the determination of the binding epitope. ${ }^{[9]}$

Interestingly, the epitope mapping indicates more intimate contacts to the aromatic ring of dopamine compared to the carbon aliphatic moiety, with proton $\mathrm{H} 5$ receiving the highest amount of saturation $\left(100^{\wedge} \%\right)$, followed by its vicinal proton H6. Saturation decreased in the aromatic $<\mathrm{Pr}>$ aliphatic chain direction, being lowest for $\mathrm{H} 8\left(35^{\wedge} \%\right)$; this suggests a binding mode that would leave the aliphatic tail partially solvent expose (but still making contact with the PG head groups), whereas the aromatic ring would establish close interactions with the hydrophobic core of the POPG bilayer. These observations at the atomic level provide new insights into the importance of the dopamine aromatic ring on binding to PG lipids and bring the first molecular-level information on the localisation and orientation of dopamine upon binding to PG-like membranes. Our STD NMR investigations therefore complement previous ITC studies that lacked atomic resolution. ${ }^{[7]}$ Overall, our results suggest that, despite the molecular recognition of dopamine being driven by the negative charge on the lipid, once association occurs, dopamine seems to reorient in order to establish morefavourable hydrophobic interactions to the lipid tails. Future modelling studies will be relevant to explain both the electrostatics and aromatic contributions on the mode of interaction better, and the STD binding epitope provided here can aid qualitative restraints for modelling.

The observations here indicate the that binding of small neurotransmitters to lipid systems might typically be within the fast-exchange regime suitable for STD NMR spectroscopy (low binding affinity, $\mathrm{mM}--\mu \mathrm{M}) .{ }^{[10]}$ Moreover, we show that saturation of the lipid signals is a convenient source of spin diffusion within macromolecular scaffolds such as MSP-assembled nanoplatforms such nanodiscs or Lipodisqs ${ }^{[11]}$ and thus represents a powerful strategy for studying lipid--small molecule interactions at atomic resolution. Importantly, lipid signals arising from monodisperse nanodisc samples are not so broad; therefore, specialised magic-angle-spinning solid-state NMR spectroscopy is not required, and 
this makes them suitable lipid platforms for solution-state NMR binding studies for ligands with exchange kinetics in the accessible range for STD NMR spectroscopy.

Additionally, our work 1$)^{\wedge \wedge}$ confirms the lack of significant interactions of dopamine to zwitterionic non-hydrogen-bond-donor POPC lipids, 2)^^shows for the first time that dopamine does not bind the zwitterionic hydrogen-bond-donor POPE lipids and, importantly, $3)^{\wedge \wedge}$ provides more-detailed information on the nature of the interactions governing ligand binding to lipids, thus using dopamine and POPG nanodiscs as a model system, compared to previous ITC studies, which were unable to provide insights at the atomic level.

\section{Experimental Section}

MSPD1 expression and purification: The membrane scaffold protein 1D1 (MSP1D1) construct was obtained from AddGene (plasmid 20061). ${ }^{[12]}$ The protein was expressed and purified according to Ritchie et ${ }^{\wedge \wedge}$ al.,${ }^{[8]}$ with modifications. Briefly, the protein was expressed in BL21(DE3) Escherichia coli cells (Calbiochem) at $37^{\wedge}{ }^{\circ} \mathrm{C}$ in $2^{\wedge \wedge}$ L flasks containing $\langle$ ? $\rangle\langle$ ? $>$ Terrific Broth $O K ?<?>\left\langle ?>\left(500^{\wedge \wedge} \mathrm{mL}\right)\right.$ until the $\mathrm{OD}_{600}$ reached 1.6. Expression was induced with isopropyl $\beta$-D-1thiogalactopyranoside (IPTG, $\left.1^{\wedge \wedge} \mathrm{mM}\right)$, and cells were harvested by centrifugation $\left(8000^{\wedge} g ; 15^{\wedge \wedge} \mathrm{min}\right.$, $\left.4^{\wedge \circ} \mathrm{C}\right)$. Cells were lysed by sonication in a buffer containing pepstatin ${ }^{\wedge} \mathrm{A}\left(2^{\wedge \wedge} \mu \mathrm{g}^{\wedge} \mathrm{mL}^{<\mathrm{M}->1}\right)$, leupeptin $\left(2^{\wedge} \mu \mathrm{g}^{\wedge} \mathrm{mL} \mathrm{L}^{<\mathrm{M}->1}\right)$ and aprotinin $\left(3^{\wedge \wedge} \mu \mathrm{g}^{\wedge} \mathrm{mL}^{<\mathrm{M}->1}\right)$. MSP1D1 was purified by $<$ ? $><?>$ please define IMAC $<$ ? $><$ ? $>$ on a nickel column. The fractions containing MSP were pooled down and dialysed against buffer $\left(20^{\wedge \wedge} \mathrm{mM}\right.$ Tris $<\mathrm{M} .>\mathrm{HCl}, \mathrm{pH}^{\wedge \wedge} 7.4,0.1^{\wedge} \mathrm{M} \mathrm{NaCl}, 0.5^{\wedge \wedge} \mathrm{mM}$ EDTA $)$ at $4{ }^{\wedge}{ }^{\circ} \mathrm{C}$. The protein sample was then filtered through a $0.22^{\wedge \wedge} \mathrm{mm}$ filter, and $0.01^{\wedge} \% \mathrm{NaN}_{3}$ was added for storage.

$<+>$ Nanodisc formation and purification: First, lipid films were formed by evaporation of lipid dissolved in chloroform $\left(1^{\wedge \wedge} \mathrm{mL}, 25^{\wedge \wedge} \mathrm{mg}^{\wedge} \mathrm{mL}^{<\mathrm{M}->1}\right)$ in a rotavapor with fast spinning for $10^{\wedge \wedge} \mathrm{min}$, then they were left in the desiccator under vacuum $\left(<10^{<\mathrm{M}->5 \wedge \wedge} \mathrm{T}\right)$ overnight. The films were then dissolved in lipid buffer $\left(50^{\wedge \wedge} \mathrm{mM}\right.$ Tris, $\mathrm{pH}^{\wedge \wedge} 7.4, \mathrm{NaCl} 100^{\wedge \wedge} \mathrm{mM}$, EDTA $1^{\wedge \wedge} \mathrm{mM}$, sodium cholate $100^{\wedge \wedge} \mathrm{mM}$ ) to a concentration of $50^{\wedge \wedge} \mathrm{mM}$, sonicated for $1^{\wedge \wedge} \mathrm{min}$, freeze-thaw three times and further sonicated for another minute.

$<+>$ Nanodiscs were assembled by adding MSP to the solubilised lipid film at a MSP-to-lipid ratio of $\left\langle ?><?>1: 70^{\prime} 1: 61^{\prime} ?<?><?>\left(241^{\wedge \wedge} \mu \mathrm{M}\right.\right.$ and $14.7^{\wedge \wedge} \mathrm{mM}$, respectively $)$. The mixture was 
incubated for $1^{\wedge \wedge} \mathrm{h}$ at $4^{\wedge}{ }^{\circ} \mathrm{C}$. To remove the sodium cholate detergent, buffer equilibrated biobeads SM$2\left(400^{\wedge} \mathrm{mg}^{\wedge} \mathrm{mL}^{<\mathrm{M}->1}\right)$ were added and the samples incubated overnight at $44^{\circ} \mathrm{C}$. Finally, the biobeads were removed by centrifugation $\left(<?>\left\langle ?>10^{\wedge} 000^{\wedge \wedge} \mathrm{rpm}\right.\right.$ please give $\mathrm{g}$ value or rotor type $<$ ? $>\langle$ ? $>$, $10^{\wedge \wedge} \min , 4^{\wedge}{ }^{\circ} \mathrm{C}$ ) and the supernatant was taken for purification by SEC on a Superdex $20010 / 300 \mathrm{GL}$ gel filtration column.

$<+>$ NMR spectroscopy: SEC-purified nanodiscs (fractions $11.5--14.5^{\wedge \wedge} \mathrm{mL}$ ) were concentrated to $300^{\wedge} \mu \mathrm{L}$ and a MSP concentration of about $70^{\wedge \wedge} \mu \mathrm{M}$ was measured from absorbance at $280^{\wedge} \mathrm{nm}$; this was equivalent to $35^{\wedge} \mu \mathrm{M}$ of nanodiscs ( 2 MSPD1 proteins per disc). The corresponding amount of nanodiscs to achieve a final concentration of $50^{\wedge \wedge} \mu \mathrm{M}$ of MSP was buffer exchanged into NMR buffer (phosphate-buffered saline, $\mathrm{pH}^{\wedge \wedge} 7.4,100^{\wedge} \% \mathrm{D}_{2} \mathrm{O}$ ) by using Vivaspin columns. A final volume of $280^{\wedge \wedge} \mu \mathrm{L}$ was used in a $5^{\wedge \wedge} \mathrm{mm}$ Shigemi tube. STD NMR experiments were carried out on a Bruker Avance^^ $\mathrm{III} 600^{\wedge \wedge} \mathrm{MHz}$ or $750^{\wedge \wedge} \mathrm{MHz}$, equipped with cryoprobe, at $20^{\wedge \circ} \mathrm{C}$. For the POPC and POPG samples, solvent suppression (Watergate) and a $15^{\wedge \wedge} \mathrm{ms}$ spin-lock filter were used after the $90^{\circ}$ pulse to reduce residual signals from the protein (MSP). No solvent suppression was used for the POPE sample. For selective lipid saturation, cascades of $49^{\wedge} \mathrm{ms}$ Gaussian-shaped pulses at a field strength of $50^{\wedge \wedge} \mathrm{Hz}$ were employed, with a delay of $1^{\wedge \wedge} \mathrm{ms}$ between successive pulses.

$<+>$ The on-resonance and off-resonance frequencies were set to 1.14 (lipid signal) and $50^{\wedge \wedge} \mathrm{ppm}$, respectively. The relaxation delay was properly adjusted so that the experiment time length was kept constant $\left(6^{\wedge \wedge} \mathrm{s}\right)$.

$<+>$ Control STD NMR experiments were carried out on a sample containing dopamine and MSP in NMR buffer, saturating the MSP signal $\left(0.74^{\wedge \wedge} \mathrm{ppm}\right)$ during the on-resonance acquisitions.

$<+>$ Epitope mapping of dopamine--POPG interactions: STD amplification factors (STD$\mathrm{AF}_{0}$ ) were calculated from the STD initial slopes. To do so, the evolution of the STD-AF with $t_{\text {sat }}$ was fitted to the monoexponential equation $\operatorname{STD}-\mathrm{AF}(t)=a(1<\mathrm{M}->\exp (<\mathrm{M}->b t))$, in which the parameter $a$ represents the asymptotic maximum of the STD build-up curve ( $\left.\mathrm{STD}^{\max }\right)$, and $b$ is the rate constant that measures the speed of the STD build-up $\left(k_{\mathrm{sat}}\right)$ and is related to the relaxation properties of a given proton. Thus, the $\mathrm{STD}-\mathrm{AF}_{0}$ values were obtained $<$ ? $><$ ? $>$ just 'as the product $a b^{\prime}$ ? $<$ ? $><?>$. ${ }^{[9]}$ 


\section{Acknowledgements}

A.W. and J.C.M.-G. acknowledge UCBCelltech for financial support under the UCBCelltech-Oxford research programme.

\section{Conflict of Interest}

The authors declare no conflict of interest. $\langle ?\rangle\langle ?\rangle$ Not even with Celltech? $<?><?\rangle$ $<$ lit1><jnl>J.^^C. Muñoz-García, E. Chabrol, R.^^R. Vivès, A. Thomas, J.^^L. de^^Paz, J. Rojo, A. Imberty, F. Fieschi, P.^^M. Nieto, J. Angulo, J. Am. Chem. Soc. 2015, 137, 4100--4110</jnl>.

$<$ lit2><jnl>J. Angulo, S.^^A. Goffin, D. Gandhi, M. Searcey, L.^^A. Howell, Chem. Eur. J. 2016, 22, 5858--5862</jnl >.

$<$ lit3 $><$ jnl $>$ T. Haselhorst, J.-M. Garcia, T. Islam, J.^^C.^^C. Lai, F.^^J. Rose, J.^^M.

Nicholls, J.^^S.^^M. Peiris, M. von^^Itzstein, Angew. Chem. Int. Ed. 2008, 47, 1910-1912; Angew. Chem. 2008, 120, 1936--1938</jnl>.

$<$ lit4><jnl $>$ S. Di^^Micco, C. Bassarello, G. Bifulco, R. Riccio, L. Gomez-Paloma, Angew. Chem. Int. Ed. 2006, 45, 224--228; Angew. Chem. 2006, 118, 230--234</jnl>.

$<$ lit5><jnl>M. Mayer, T.^^L. James, J. Am. Chem. Soc. 2004, 126, 4453--4460</jnl>. $<$ lit6><jnl>F.^^M. Assadi-Porter, M. Tonelli, E. ${ }^{\wedge}$ L. Maillet, J.^^L. Markley, M. Max, Biochim. Biophys. Acta Biomembr. 2010, 1798, 82--86</jnl>.

$<$ lit7><jnl>K. Jodko-Piorecka, G. Litwinienko, ACS Chem. Neurosci. 2013, 4, 1114-$1122</$ jnl $>$.

$<$ lit8 $><$ book $>$ T.^^K. Ritchie, Y..$^{\wedge}$ V. Grinkova, T.^^H. Bayburt, I. ${ }^{\wedge}$ G. Denisov, J.^^K. Zolnerciks, W.^^M. Atkins, S.^^G. Sligar, Methods in Enzymology, Vol. 464 (Ed.: D. Nejat), Academic Press, San Diego, 2009, pp.^^211--231</book>. $<$ lit9><jnl >J. Angulo, P.^^M. Enríquez-Navas, P.^^M. Nieto, Chem. Eur. J. 2010, 16, 7803-$7812</$ jnl $>$.

$<$ lit10><jnl $>$ V.^^V. Krishnan, Curr. Anal. Chem. 2005, 1, 307--320</jnl>. 
$<$ lit11 $><$ jnl $>$ M.^^C. Orwick, P.^^J. Judge, J. Procek, L. Lindholm, A. Graziadei, A. Engel, G.

Gröbner, A. Watts, Angew. Chem. Int. Ed. 2012, 51, 4653--4657; Angew. Chem. 2012, $124,4731--4735</ j n l>$.

$<$ lit12><jnl>I.^^G. Denisov, Y.^^V. Grinkova, A. ${ }^{\wedge \wedge}$ A. Lazarides, S. ${ }^{\wedge}$ G. Sligar, J. Am. Chem. Soc. 2004, 126, 3477--3487</jnl>.

Manuscript received: February 7, 2018

Accepted manuscript online: March 14, 2018

Version of record online: $\langle$ ? $>\langle$ ? $>$

Figure $^{\wedge \wedge} 1 \quad$ SEC profile (Superdex 200 10/300 GL gel filtration column) of POPC$(<$ col $=\{100,100,0,0\}\rangle\langle\mathbf{L}-></$ col $>)$ and POPG-only $(\langle$ col $=\{0,100,100,0\}\rangle\langle\mathbf{L}-\rangle</$ col $\rangle)$ nanodiscs.

Figure $^{\wedge} 2$ STD NMR spectra of $25^{\wedge \wedge} \mu \mathrm{M}$ POPC $(<\operatorname{col}=\{100,100,0,0\}><\mathrm{L}-></$ col $>)$ and POPG $(<\mathrm{col}=\{0,100,100,0\}><\mathrm{L}-></ \mathrm{col}>)$ nanodiscs in the presence of $1^{\wedge \wedge} \mathrm{mM}$ dopamine $\left(600^{\wedge \wedge} \mathrm{MHz}, 20^{\wedge}{ }^{\circ} \mathrm{C}, 4^{\wedge \wedge} \mathrm{S}\right.$ saturation time). The on-resonance and off-resonance frequencies were set to 1.14 (lipid signal) and $50^{\wedge \wedge} \mathrm{ppm}$, respectively. The inset shows the aromatic protons of dopamine.

Figure $^{\wedge \wedge}$ Reference $(<$ col $=\{100,100,0,0\}\rangle\langle\mathbf{L}-></$ col $>)$ and STD $(<\mathrm{col}=\{0,100,100,0\}><\mathrm{L}-></ \mathrm{col}>, \times 16)$ spectra of $25^{\wedge \wedge} \mu \mathrm{M}$ POPE nanodiscs in the presence of $1^{\wedge \wedge} \mathrm{mM}$ dopamine $\left(750^{\wedge \wedge} \mathrm{MHz}, 20^{\wedge} \mathrm{C}, 4^{\wedge \wedge} \mathrm{s}\right.$ saturation time). The on-resonance and offresonance frequencies were set to 1.14 (lipid signal) and $50^{\wedge \wedge} \mathrm{ppm}$, respectively.

Figure $\left.{ }^{\wedge} 4 \quad \mathrm{~A}\right)^{\wedge \wedge} \mathrm{STD}$ build-up curves of $1^{\wedge \wedge} \mathrm{mM}$ dopamine in the presence of $25^{\wedge \wedge} \mu \mathrm{M}$ POPG nanodiscs. A $)^{\wedge \wedge}\langle$ col $=\{0,100,100,0\}>\langle$ ?qv $>\langle/$ col $>$ : H2, $\langle$ col $=\{100,0,100,0\}\rangle\langle$ ?kv $>\langle/$ col $>$ : H5, $\langle$ col $=\{100,100,0,0\}\rangle\langle$ ?dov $>\langle/$ col $>$ : H6, $\langle$ col $=\{0,20,100,0\}\rangle\langle$ ?ql $>\langle/$ col $>:$ H7, $\langle$ ?kl $>$ : H8. B $)^{\wedge \wedge}$ Binding epitope of dopamine derived from the initial slopes of the STD NMR buildup curves. The numbers denote the fraction of saturation received from the POPG nanodiscs by dopamine protons, relative to the maximum STD signal $\left(\mathrm{H} 5,100^{\wedge} \%\right)$. Values close to 
$100^{\wedge} \%$ represent the most intimate contacts to the membrane surface in the bound state. $<$ ? $><$ ? Significance of red and blue? $<$ ? $><?>$ 